\title{
Revisão
}

\section{Instrumentos para avaliação de habilidades de comunicação no cuidado em saúde no Brasil: uma revisẩo de escopo*}

Instruments for assessing communication skills in the area of healthcare in Brazil: a scoping review (abstract: p. 13)

Instrumentos para evaluación de habilidades de comunicación en el cuidado de salud en Brasil: una revisión de alcance (resumen: p. 13)

Dyego Carlos Souza Anacleto de Araújo(a)

<dyegodm_pb@hotmail.com>

Pedro Wlisses dos Santos Menezes ${ }^{(b)}$

<pedrowwwmenezes@gmail.com> iD

Afonso Miguel das Neves Cavaco ${ }^{(c)}$

<acavaco@ff.ulisboa.pt>

Alessandra Rezende Mesquita ${ }^{(\mathrm{d})}$

<alessandra_pharmacia@hotmail.com>

Divaldo Pereira de Lyra Júnior ${ }^{(e)}$

<lepfs.ufs@gmail.com> iD

\author{
* Este estudo integra o projeto \\ de doutorado do primeiro autor \\ bolsista da Coordenação de \\ Aperfeiçoamento de Pessoal \\ de Nível Superior (Capes). \\ (a) Pós-graduando do Programa \\ de Pós-Graduação em Ciências \\ da Saúde (Doutorado), \\ Laboratório de Ensino e \\ Pesquisa em Farmácia Social \\ (LEPFS), Universidade Federal \\ de Sergipe (UFS). Avenida \\ Marechal Rondon, $s / n$., Jardim \\ Rosa Elze. São Cristóvão, \\ Sergipe (SE), Brasil. 49100-000. \\ (b) Graduando do curso de \\ Farmácia, LEPFS, UFS. São \\ Cristóvão, SE, Brasil. \\ continua pág. 10
}

O ensino de habilidades de comunicação é indispensável aos profissionais de saúde. Este estudo objetivou identificar instrumentos validados no português do Brasil que avaliam habilidades de comunicação de estudantes ou profissionais da saúde. Para tanto, foi realizada uma revisão de escopo que incluiu estudos de validação de instrumentos para avaliação de habilidades de comunicação de estudantes/profissionais da área da Saúde. Foram identificados quatro instrumentos, traduzidos e validados para o português do Brasil. Validação de conteúdo, validação de construto e o teste de consistência interna foram realizados com maior frequência. Esta revisão identificou um número reduzido de instrumentos para avaliar habilidades de comunicação e dados limitados de validade e confiabilidade dos instrumentos.

Palavras-chave: Estudos de validação. Habilidades de comunicação. Cuidado em saúde.

Araújo DCSA, Menezes PWS, Cavaco AMN, Mesquita AR, Lyra-Jr DP. Instrumentos para avaliação de habilidades de comunicação no cuidado em saúde no Brasil: uma revisão de escopo. Interface (Botucatu). 2020; 24: e200030 https://doi.org/10.1590/Interface.200030 


\section{Introdução}

A comunicação é uma habilidade indispensável aos profissionais de saúde por possibilitar o acolhimento adequado das necessidades do paciente e de seus familiares, construir relações de confiança, aumentar a satisfação e melhorar a compreensão da doença e do tratamento pelos pacientes ${ }^{1-3}$. Por isso, o ensino de habilidades de comunicação passou a ser recomendado em diretrizes curriculares de cursos de graduação da área da Saúde, em todo o mundo ${ }^{4-9}$.

No Brasil, após a publicação da primeira resolução com diretrizes curriculares para cursos da área da Saúde, em 2001, as habilidades de comunicação passaram a figurar como uma das cinco competências gerais a serem desenvolvidas pelos profissionais de saúde. De acordo com essas diretrizes, os profissionais devem ser acessíveis e manter a confidencialidade das informações recebidas, na interação com outros profissionais de saúde e pacientes ${ }^{10}$. Apesar de sua importância, estudos realizados em cursos de graduação em Medicina e Farmácia no Brasil reportam que ainda persistem lacunas curriculares para o desenvolvimento dessa habilidade ${ }^{11,12}$.

O desenvolvimento de habilidades de comunicação depende, sobretudo, da inserção curricular e repetição de atividades práticas pelos estudantes. Por isso, além da definição dos objetivos de aprendizagem e da inserção do ensino da comunicação nos programas curriculares, é necessário definir métodos e estratégias de ensino e avaliação adequadas ${ }^{6,13}$. A literatura tem destacado que a integração de aulas expositivas, discussóes, role-play, pacientes simulados e simulaçốes por computador têm sido efetivas no aprendizado de habilidades de comunicação ${ }^{14-19}$. Entretanto, tão importante quanto ensinar é avaliar a efetividade da aprendizagem, possibilitando conhecer os aspectos da comunicação que precisam ser melhorados e fornecendo feedback adequado ao estudante.

A avaliação das habilidades de comunicação pode ser realizada de diversas maneiras e requer, na maioria das vezes, o uso de instrumentos adequados de mensuração para autoavaliação e/ou observação direta por terceiros. Para tanto, são necessários instrumentos validados para avaliação das habilidades de comunicação, de modo a fornecer dados confiáveis e consistentes para os envolvidos no processo ensino-aprendizagem. Nesse contexto, o objetivo desta revisão de escopo foi identificar instrumentos para avaliação de habilidades de comunicação, em português do Brasil, com evidências de validade para estudantes e/ou profissionais da saúde.

\section{Metodologia}

Uma revisão de escopo da literatura foi realizada com a finalidade de responder a seguinte pergunta: qual(is) é(são) o(s) instrumento(s) em português do Brasil para avaliação de habilidades de comunicação com evidências de validade e confiabilidade para estudantes/profissionais da área da saúde? 


\section{Estratégia de busca}

As buscas foram realizadas nas das seguintes bases de dados: SciELO, Latin American and Caribbean Center on Health Sciences Information (Lilacs), Index psicologia, PubMed, Education Resources Information Center (Eric) e Web of Science. A estratégia de busca foi adaptada de acordo com os protocolos de cada base, utilizando a combinação dos seguintes descritores: communication AND instrument $A N D$ validity study $A N D$ Brazil. As palavras-chaves foram definidas utilizando o vocabulário controlado da Medical Subject Headings (MeSH). Termos não padronizados também foram utilizados para expandir a estratégia de busca. Busca por literatura cinzenta foi realizada no Banco de Teses e Dissertaçôes da Capes e e-mails foram enviados aos pesquisadores. A estratégia de busca completa se encontra descrita no Quadro 1.

Quadro 1. Estratégia de busca

\begin{tabular}{|c|c|c|}
\hline Base de dados & Estratégia & $\mathrm{N}^{0}$ de estudos \\
\hline SciELO & 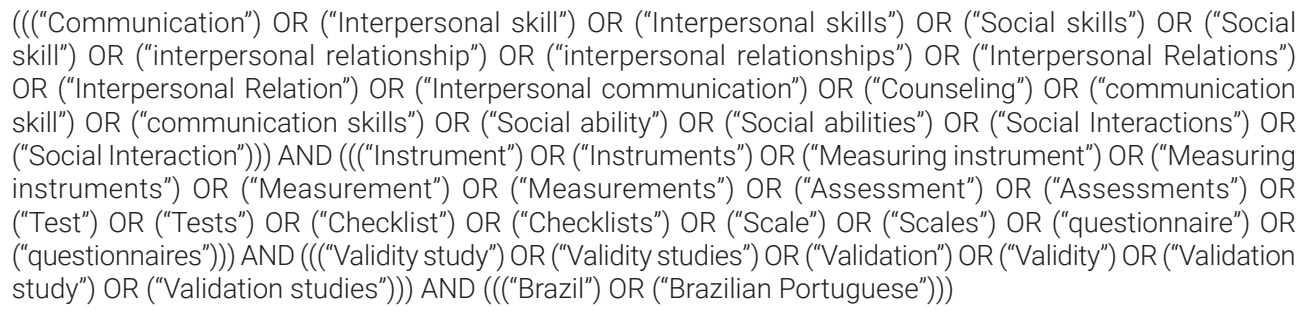 & 40 \\
\hline LILACS & 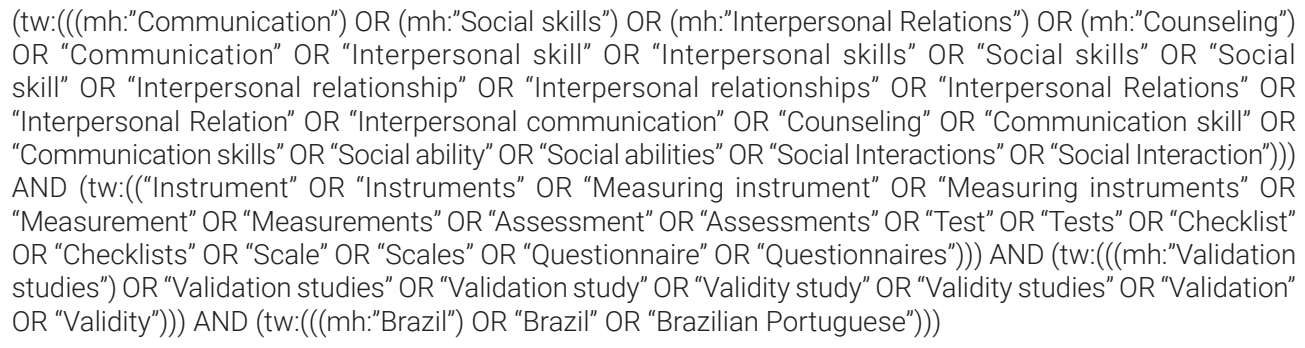 & 83 \\
\hline Index Psicologia & 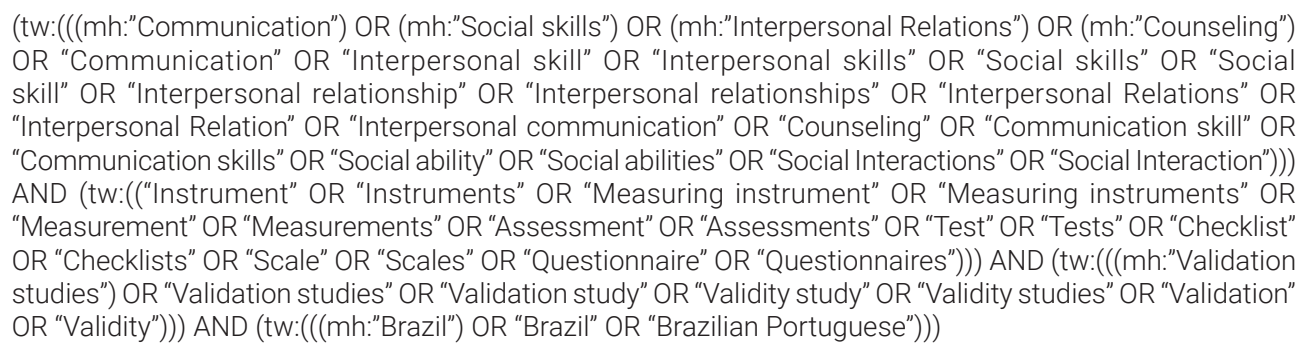 & 21 \\
\hline PUBMED* & 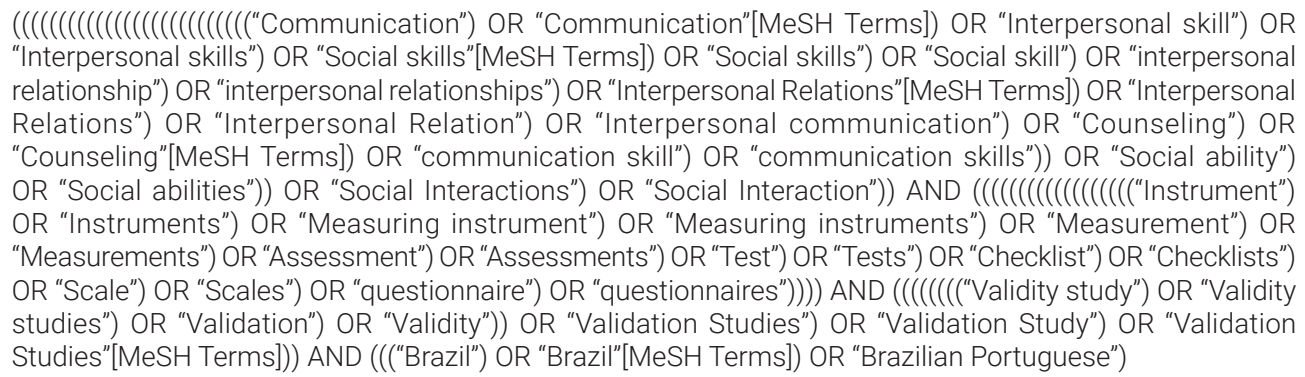 & 483 \\
\hline
\end{tabular}




\begin{tabular}{|c|c|c|}
\hline Base de dados & Estratégia & $\mathrm{N}^{\circ}$ de estudos \\
\hline ERIC & 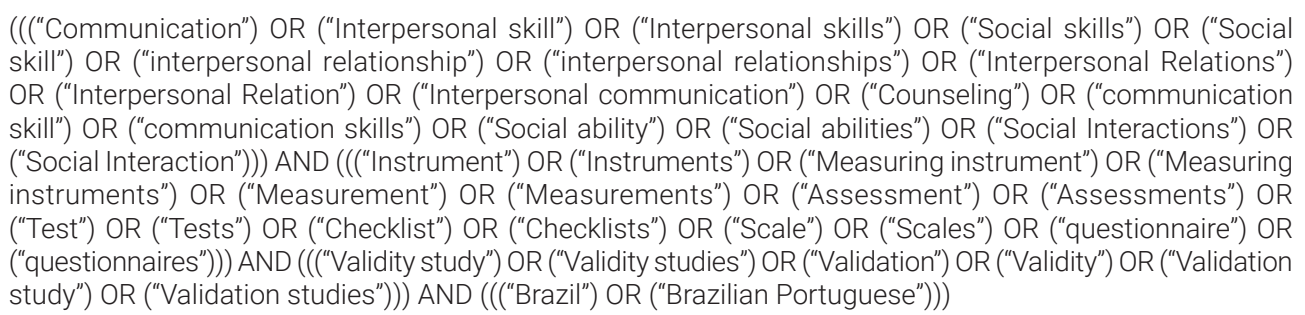 & 4 \\
\hline Web of Science & 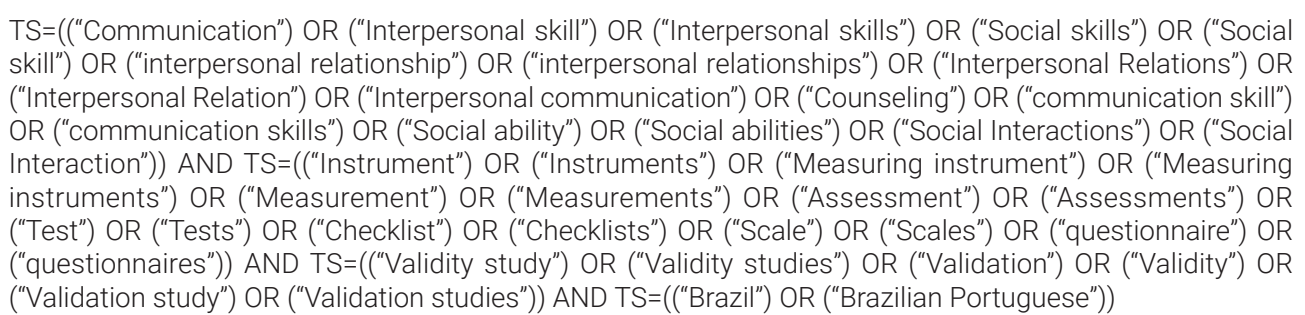 & 71 \\
\hline
\end{tabular}

\section{Critérios de inclusão e exclusão}

Foram incluídos estudos publicados em qualquer idioma até fevereiro de 2019 que atenderam aos seguintes critérios: (i) estudo de validação de instrumento para português (Brasil); o qual (ii) avalia habilidades de comunicação e (iii) tem profissionais e/ou estudantes da área da saúde como público-alvo. Foram excluídos os estudos não disponíveis na íntegra, cuja solicitação de arquivo não foi respondida pelo autor e publicaçôes em congressos.

\section{Seleção dos artigos e extração dos dados}

A seleção dos estudos foi realizada utilizando plataforma on-line para revisóes sistemáticas Rayyan QCRI ${ }^{20}$. Estudos em duplicata foram excluídos. A triagem de títulos, resumos e texto completo e extração de dados foram realizadas por dois avaliadores de forma independente e as divergências foram resolvidas por um terceiro revisor. Foram extraídos: autores, ano de publicação, denominação do instrumento, país de origem, amostra, tipo de avaliação (autoavaliação ou observação direta) tipo de escala, seçôes/domínios e evidências de validade e fidedignidade avaliadas.

\section{Avaliação da qualidade de estudos de adaptação transcultural}

Quando os instrumentos identificados haviam sido submetidos à adaptação e validação transcultural, o estudo foi avaliado de acordo com as Diretrizes de Adaptação Transcultural de Questionários ${ }^{21}$. A qualidade das etapas foi classificada como positiva (+), quando realizada conforme procedimento adotado; duvidosa (?), quando realizado de maneira questionável; negativa (-), quando o procedimento foi realizado corretamente, mas com número insuficiente de tradutores; ou nula(0), quando não havia informações suficientes para qualificar a etapa. 


\section{Resultados}

Foram identificadas 702 publicaçốes científicas nas bases de dados. No Banco de Teses e Dissertações da Capes, foram identificados três estudos. Após a leitura de títulos, resumos e textos completos, quatro instrumentos foram incluídos na revisão: "Avaliação do Processo de Atendimento Farmacêutico"22, "Escala de Competência em Comunicação Interpessoal"23, "Formulário de Avaliação de Habilidades de Comunicação GapKalamazoo" ${ }^{24}$ e "Autoavaliação sobre profissionalismo e comunicação interpessoal entre enfermeiros e paciente" 25 . O processo de seleção dos artigos se encontra descrito na figura 1.

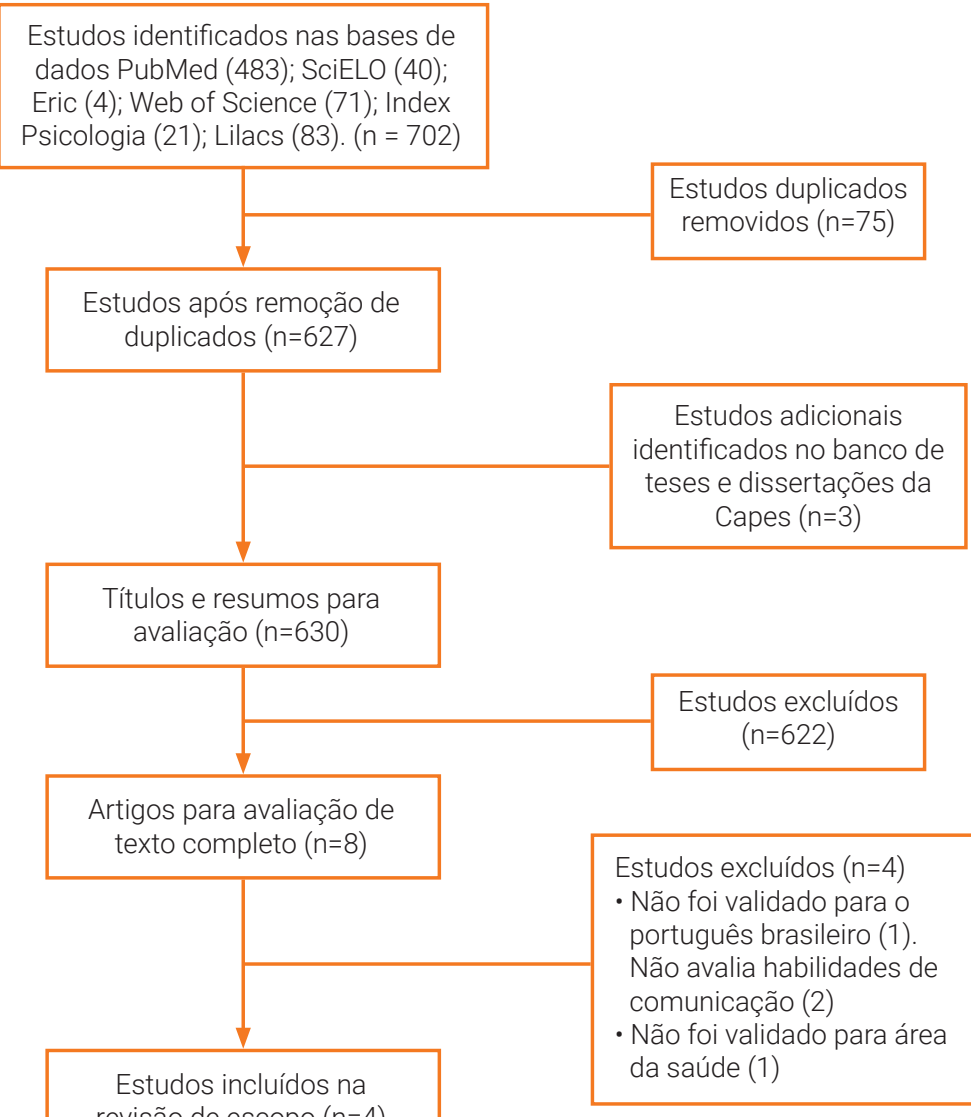

Figura 1. Descrição do processo de seleção dos artigos.

Fonte: os autores.

Todos os estudos foram publicados entre os anos de 2012 e 2017 e consistiram em validações de instrumentos, desenvolvidos na Alemanha e nos Estados Unidos, que passaram por tradução e adaptação transcultural para o português do Brasil, seguidos por análise de evidências de validade para estudantes de Farmácia ${ }^{22}$ e de Enfermagem ${ }^{23}$; enfermeiros ${ }^{25}$; e médicos ${ }^{24}$. Quanto ao tipo de avaliação, os instrumentos são destinados à autoavaliação $(2)^{23,25}$, avaliação por observação direta $(1)^{22}$ e avaliação do tipo 360 graus $(1)^{24}$. Todos os estudos utilizaram de escalas Likert de cinco pontos para avaliação dos itens. As características gerais dos instrumentos se encontram descritas na tabela 1. 
Tabela 1. Características dos instrumentos para avaliação de habilidades de comunicação validados para o português do Brasil

\begin{tabular}{|c|c|c|c|c|c|}
\hline Autor & $\begin{array}{c}\text { Instrumento } \\
\text { [país de origem] }\end{array}$ & Tipo de avaliação & Domínios/seções & Amostra & Avaliação \\
\hline $\begin{array}{l}\text { Mesquita et al., } \\
\text { 2012* }\end{array}$ & $\begin{array}{l}\text { Avaliação do Processo } \\
\text { de Atendimento } \\
\text { Farmacêutico } \\
\text { [Alemanha] }\end{array}$ & $\begin{array}{l}\text { Escala Likert de cinco } \\
\text { pontos e questões do } \\
\text { tipo "sim ou não" }\end{array}$ & $\begin{array}{l}\text { Comunicação não verbal; } \\
\text { compreensibilidade da orientação; } \\
\text { impressão geral da orientação }\end{array}$ & $\begin{array}{l}\text { Estudantes de } \\
\text { Farmácia }\end{array}$ & $\begin{array}{l}\text { Observação } \\
\text { direta }\end{array}$ \\
\hline $\begin{array}{l}\text { Puggina; Silva, } \\
2014\end{array}$ & $\begin{array}{l}\text { Escala de Competência } \\
\text { em Comunicação } \\
\text { Interpessoal } \\
\text { [Estados Unidos] }\end{array}$ & $\begin{array}{c}\text { Escala Likert de cinco } \\
\text { pontos }\end{array}$ & $\begin{array}{l}\text { Controle do ambiente; autorrevelação; } \\
\text { assertividade; Manejo das interações; } \\
\text { disponibilidade }\end{array}$ & $\begin{array}{l}\text { Estudantes de } \\
\text { Enfermagem }\end{array}$ & Autoavaliação \\
\hline $\begin{array}{l}\text { Amaral et al., } \\
2016\end{array}$ & $\begin{array}{l}\text { Formulário de Avaliação } \\
\text { de Habilidades de } \\
\text { Comunicação Gap- } \\
\text { Kalamazoo } \\
\text { [Estados Unidos] }\end{array}$ & $\begin{array}{c}\text { Escala Likert de cinco } \\
\text { pontos }\end{array}$ & $\begin{array}{l}\text { Construção de relação; abertura } \\
\text { da discussão; recolhimento de } \\
\text { informações; compreensão da } \\
\text { perspectiva do paciente e da família; } \\
\text { compartilhamento de informações; } \\
\text { estabelecimento de acordos; } \\
\text { conclusão do diálogo; demonstração } \\
\text { de empatia; transmissão de } \\
\text { informações precisas }\end{array}$ & Médicos & $\begin{array}{l}\text { Observação } \\
\text { direta; } \\
\text { autoavaliação; } \\
\text { avaliação por } \\
\text { familiares }\end{array}$ \\
\hline $\begin{array}{c}\text { Pereira; } \\
\text { Puggina, } 2017\end{array}$ & $\begin{array}{l}\text { Autoavaliação sobre } \\
\text { profissionalismo e } \\
\text { comunicação interpessoal } \\
\text { entre enfermeiros e } \\
\text { paciente } \\
\text { [Estados Unidos] }\end{array}$ & $\begin{array}{l}\text { Escala Likert de } \\
\text { cinco pontos }\end{array}$ & $\begin{array}{l}\text { Habilidade interpessoal; troca de } \\
\text { informações; sinceridade na relação; } \\
\text { profissionalismo }\end{array}$ & Enfermeiros & Autoavaliação \\
\hline
\end{tabular}

*Este instrumento é composto por três seções. As informações utilizadas nesta revisão se referem apenas à seção referente a habilidades de comunicação.

O processo de adaptação transcultural de dois instrumentos atendeu às diretrizes adotadas por esta revisão $0^{23,25}$. Todos instrumentos passaram por validação de conteúdo e, em três desses, foi realizada validação de construto ${ }^{23-25}$. Avaliação da consistência interna foi o teste de fidedignidade realizado com maior frequência ${ }^{23-25}$. A tabela 2 apresenta informações sobre adaptação transcultural e os tipos de validação identificados nos estudos. 
Tabela 2. Características dos instrumentos para avaliação de habilidades de comunicação validados para o português do Brasil

\begin{tabular}{|c|c|c|c|c|c|c|c|c|c|c|}
\hline \multirow[b]{2}{*}{ Instrumento } & \multicolumn{4}{|c|}{ Adaptação transcultural } & \multirow[b]{2}{*}{ Pré-teste } & \multirow[b]{2}{*}{$\begin{array}{l}\text { Validade } \\
\text { conteúdo }\end{array}$} & \multirow[b]{2}{*}{$\begin{array}{l}\text { Validade } \\
\text { construto }\end{array}$} & \multicolumn{3}{|c|}{ Fidedignidade } \\
\hline & Tradução & Síntese & Retrotradução & $\begin{array}{c}\text { Revisão do } \\
\text { comitê de } \\
\text { especialistas }\end{array}$ & & & & Estabilidade & Equivalência & $\begin{array}{c}\text { Consistência } \\
\text { interna }\end{array}$ \\
\hline $\begin{array}{l}\text { Avaliação do Processo de } \\
\text { Atendimento Farmacêutico } \\
\text { (Mesquita et al., 2012) }\end{array}$ & + & + & - & + & + & $\operatorname{Sim}$ & Não & Não & Não & Não \\
\hline $\begin{array}{c}\text { Escala de Competência em } \\
\text { Comunicação Interpessoal } \\
\text { (Puggina; Silva, 2014) }\end{array}$ & + & + & + & + & + & Sim & Sim & Não & Não & $\operatorname{Sim}$ \\
\hline $\begin{array}{l}\text { Formulário de avaliação de } \\
\text { habilidades de Comunicação } \\
\text { Gap-Kalamazoo } \\
\text { (Amaral et al., 2016) }\end{array}$ & + & + & - & + & + & $\operatorname{Sim}$ & $\operatorname{Sim}$ & $\operatorname{Sim}$ & Sim & $\operatorname{Sim}$ \\
\hline $\begin{array}{c}\text { Autoavaliação sobre } \\
\text { profissionalismo e } \\
\text { comunicação interpessoal } \\
\text { entre enfermeiros e paciente } \\
\text { (Pereira; Puggina, 2017) }\end{array}$ & + & + & + & + & + & Sim & $\operatorname{Sim}$ & Não & Não & Sim \\
\hline
\end{tabular}

\section{Discussão}

Um dos achados mais importantes desta revisão sistemática está relacionado ao número reduzido de instrumentos que avaliam habilidades de comunicação na área da Saúde com evidências de validade para o português, sugerindo que, apesar de sua importância, a comunicação ainda é um tema pouco discutido e estudado no Brasil. Além disso, o fato de os estudos terem sido publicados nesta década demonstra recente interesse pelo uso de instrumentos com evidências de validade para avaliar a comunicação. Isso pode estar associado a novas diretrizes educacionais e políticas públicas nacionais que orientam a formação e atuação de profissionais, aptos a oferecer atendimento integral e humanizado nos sistemas de saúde ${ }^{26-28}$.

Em outros países, como Estados Unidos, Alemanha e Reino Unido, pesquisas relacionadas ao ensino e avaliação de habilidades de comunicação na área da saúde são bem consolidadas e a literatura contempla uma série de instrumentos desenvolvidos e validados para essa finalidade ${ }^{29-31}$.Nesta revisão, não foram identificados instrumentos 
de origem brasileira, ou seja, todos foram traduzidos e adaptados de outros países. A adaptação transcultural e validação de instrumentos tem se tornado uma prática frequente em todo o mundo, especialmente por ser um método mais barato e rápido, quando comparado ao desenvolvimento de um novo instrumento, além de possibilitar comparações com resultados de outros países ${ }^{32,33}$

A comunicação é uma habilidade complexa e de difícil avaliação; por isso, às vezes, exige o uso de múltiplos instrumentos, que possibilitem a autoavaliação ou avaliação por meio da observação direta, a depender dos objetivos propostos. Os instrumentos de autoavaliação são ferramentas importantes na consolidação da aprendizagem autodirigida, pois possibilitam ao estudante ou profissional identificar suas dificuldades e estabelecer meios de saná-las ${ }^{34}$. Por isso, organizaçóes educacionais têm recomendado que as instituiçóes de ensino encorajem e auxiliem os estudantes a assumirem a responsabilidade pela avaliação de suas próprias competências.

É importante destacar, no entanto, que autoavaliações são influenciadas pela capacidade crítica e reflexiva de quem se autoavalia, por isso, o resultado pode não corresponder à realidade. Estudo realizado por Lundquist et al..$^{35}$ constatou que os resultados da autoavaliação das habilidades de comunicação de estudantes de Farmácia foram consistentemente inferiores às avaliações dos docentes. Dessa forma, além de possibilitar o acesso a instrumentos de autoavaliação, é necessário desenvolver o senso crítico de estudantes e profissionais para que possam utilizá-los adequadamente, de modo a evitar que as habilidades sejam subestimadas ou superestimadas.

O estudo de Amaral et al. trouxe uma modalidade de avaliação que, nos últimos anos, tem sido incorporada ao processo ensino-aprendizagem: feedback em 360 graus ou feedback por múltiplas fontes, considerando a autoavaliação, a avaliação por observação direta pelo instrutor e avaliação pelo próprio paciente. A vantagem de se utilizar essa metodologia é que diferentes avaliadores se concentram em avaliar características específicas e, conjuntamente, fornecem uma avaliação mais abrangente quando comparada àquela realizada de forma isolada. Revisão sistemática realizada por Donnon et al..$^{36}$ demonstrou que esse tipo de feedback apresentou confiabilidade e validade na avaliação da prática médica. Dessa forma, a incorporação da avaliação por diferentes atores, embora necessite de mais recursos, poderá possibilitar uma análise mais complexa e precisa das habilidades de comunicação de estudantes ou profissionais de saúde.

Tanto o instrumento traduzido e validado por Amaral et al. ${ }^{24}$ quanto o traduzido e avaliado por Mesquita et al. ${ }^{22}$ possibilitam a avaliação de habilidades de comunicação em situações simuladas. As simulações têm se destacado na avaliação de competências clínicas de estudantes da área da saúde e, especialmente, do processo ensino-aprendizagem da comunicação, pois possibilitam avaliar as interaçóes entre profissional/estudante da área da saúde e paciente em uma situação semelhante à realidade ${ }^{15,37,38}$. Os instrumentos possibilitam, assim, identificar aspectos da comunicação com paciente que precisam ser melhorados, fornecendo feedback adequado ao indivíduo avaliado.

Nesta revisão, os instrumentos apresentaram, em sua maioria, evidências de validade de conteúdo, construto e consistência interna. Semelhante aos dados deste estudo, revisão sistemática realizada por Rehim et al. ${ }^{39}$ identificou dados 
limitados de validade e confiabilidade de instrumentos para avaliar habilidades de comunicação de profissionais de saúde em hospitais. Cömert et al. ${ }^{29}$, em sua revisão sistemática, também identificaram lacunas metodológicas no relato de propriedades psicométricas de instrumentos utilizados para avaliar habilidade de comunicação em exames clínicos objetivos estruturados.

Entre os instrumentos identificados nesta revisão, o estudo de Amaral et al. ${ }^{24}$ realizou maior variedade de validaçôes, enquanto o estudo de Mesquita et al..$^{22}$ esteve restrito à validação de conteúdo. Evidências de validade e fidedignidade são fatores importantes para qualidade dos instrumentos, pois minimizam os riscos de julgamentos subjetivos do avaliador e reforçam a legitimidade e credibilidade dos resultados ${ }^{40,41}$. Por isso, recomendase que sejam realizados múltiplos tipos de validações para um mesmo instrumento, de modo a acumular evidências que corroboram a interpretação dos dados obtidos.

Este é o primeiro estudo que avalia a disponibilidade de instrumentos com evidências de validade para avaliação de habilidades de comunicação no Brasil por meio de uma busca e análise sistemática da literatura. A ausência de resposta aos e-mails enviados pelos autores solicitando teses e dissertaçôes impossibilitou a análise da literatura cinzenta, apresentando-se como uma das limitações deste estudo. Apesar da pertinência dos dados, é válido destacar que a revisão sistemática depende de publicações científicas disponíveis nas bases de dados. Eventualmente, instrumentos validados e utilizados na prática educacional podem não estar disponíveis na literatura e, por isso, não integraram esta revisão sistemática.

\section{Considerações finais}

Este estudo identificou um número reduzido de instrumentos validados para avaliação de habilidades de comunicação. Apesar da existência de inúmeros tipos de cursos da área da saúde no Brasil, apenas Enfermagem, Medicina e Farmácia foram alvos de validação de instrumentos para essa prática. Além disso, foram identificados dados limitados de validade e confiabilidade dos instrumentos.

É pertinente destacar que os resultados desta revisão suscitaram algumas reflexôes entre os pesquisadores. Embora não tenha sido objetivo deste estudo responder as seguintes questôes, estas merecem ser pontuadas: i) As habilidades de comunicação têm sido ensinadas e avaliadas durante o processo de formação de estudantes e profissionais da área da saúde no Brasil?; ii) Quais instrumentos têm sido utilizados?; e iii) Quais avanços no processo avaliativo dessa competência ainda são necessários no país?. Esses questionamentos deverão integrar uma agenda de futuros estudos na área, de modo a traçar um panorama do ensino-avaliação de habilidades de comunicação nos cursos de graduação em saúde no Brasil. 


\section{Filiação}

(c) Departamento de Farmácia Social, Universidade de Lisboa. Lisboa, Portugal.

(d) Departamento de Farmácia, LEPFS, UFS. São Cristóvão, SE, Brasil.

(e) Programa de Pós-Graduação em Ciências da Saúde, LEPFS, UFS. São Cristóvão,

SE, Brasil.

\section{Contribuições dos autores}

Todos os autores participaram ativamente de todas as etapas de elaboração do manuscrito.

\section{Direitos autorais}

Este artigo está licenciado sob a Licença Internacional Creative Commons 4.0, tipo BY (https://creativecommons.org/licenses/by/4.0/deed.pt_BR).

\section{(cc) BY}

\section{Referências}

1. Mullan BA, Kothe EJ. Evaluating a nursing communication skills training course: the relationships between self-rated ability, satisfaction, and actual performance. Nurse Educ Pract. 2010; 10(6):374-8.

2. Boissy A, Windover AK, Bokar D, Karafa M, Neuendorf K, Frankel RM, et al. Communication skills training for physicians improves patient satisfaction. J Gen Intern Med. 2016; 31(7):755-61.

3. Ranjan P, Kumari A, Chakrawarty A. How can doctors improve their communication skills? J Clin Diagn Res. 2015; 9(3):JE01-4.

4. Makoul G. The SEGUE Framework for teaching and assessing communication skills. Patient Educ Couns. 2001; 45(1):23-34.

5. American Association of Critical-Care Nurses. AACN standards for establishing and sustaining healthy work environments: a journey to excellence. Am J Crit Care. 2005; 14(3):187-97.

6. Bachmann C, Abramovitch H, Barbu CG, Cavaco AM, Elorza RD, Haak R, et al. A European consensus on learning objectives for a core communication curriculum in health care professions. Patient Educ Couns. 2013; 93(1):18-26.

7. Kitson A, Marshall A, Bassett K, Zeitz K. What are the core elements of patientcentred care? A narrative review and synthesis of the literature from health policy, medicine and nursing. J Adv Nurs. 2013; 69(1):4-15.

8. Von Fragstein M, Silverman J, Cushing A, Quilligan S, Salisbury H, Wiskin C. UK consensus statement on the content of communication curricula in undergraduate medical education. Med Educ. 2008; 42(11):1100-7. 
9. Kurtz SM, Cooke LJ. Learner-centred communication training. In: Kissane DW, Bultz BD, Butow PN, Bylund CL, Noble S, Wilkinson S, editores. Oxford textbook for communication in oncology and palliative care. Oxford: Oxford University Press; 2017.

10. Brasil. Ministério da Educação. Conselho Nacional de Educação. Câmara da Educação Superior. Parecer CNE/CES no 1.133, de 7 de Agosto de 2001. Estabelece as diretrizes curriculares para os cursos de ciências biológicas. Diário Oficial da União [Internet]. 3 Out 2001 [citado 27 Mar 2020]. Sec. 1, p. 131. Disponível em: http://portal.mec.gov. $\mathrm{br} / \mathrm{dmdocuments/ces1133.pdf}$

11. Araújo DC, Santos JS, Barros IM, Cavaco AM, Mesquita AR, Lyra DP.

Communication skills in brazilian pharmaceutical education: a documentary analysis. Pharm Pract (Granada). 2019; 17(1):1395.

12. Liberali R, Novack D, Duke P, Grosseman S. Communication skills teaching in Brazilian medical schools: What lessons can be learned? Patient Educ Couns. 2018; 101(8):1496-9.

13. García de Leonardo C, Ruiz-Moral R, Caballero F, Cavaco A, Moore P, Dupuy LP, et al. A Latin American, Portuguese and Spanish consensus on a core communication curriculum for undergraduate medical education. BMC Med Educ. 2016; 16(1):99.

14. De Marco MA, Vessoni AL, Capelo A, Dias CC. Laboratório de comunicação: ampliando as habilidades do estudante de medicina para a prática da entrevista. Interface (Botucatu). 2010; 14(32):217-27.

15. Mesquita AR, Lyra DP, Brito GC, Balisa-Rocha BJ, Aguiar PM, Almeida Neto AC. Developing communication skills in pharmacy: a systematic review of the use of simulated patient methods. Patient Educ Couns. 2010; 78(2):143-8.

16. Berkhof M, van Rijssen HJ, Schellart AJM, Anema JR, van der Beek AJ. Effective training strategies for teaching communication skills to physicians: an overview of systematic reviews. Patient Educ Couns. 2011; 84(2):152-62.

17. Kron FW, Fetters MD, Scerbo MW, White CB, Lypson ML, Padilla MA, et al. Using a computer simulation for teaching communication skills: a blinded multisite mixed methods randomized controlled trial. Patient Educ Couns. 2017; 100(4):748-59.

18. Wallman A, Vaudan C, Kälvemark Sporrong S. Communications training in pharmacy education, 1995-2010. Am J Pharm Educ. 2013; 77(2):36.

19. Liberali $\mathrm{R}$, Grosseman $S$. Use of psychodrama in medicine in Brazil: a review of the literature. Interface (Botucatu). 2015; 19(54):561-71.

20. Ouzzani M, Hammady H, Fedorowicz Z, Elmagarmid A. Rayyan-a web and mobile app for systematic reviews. Syst Rev. 2016; 5(1):1-10.

21. Beaton DE, Bombardier C, Guillemin F, Ferraz MB. Guidelines for the process of cross-cultural adaptation of self-report measures. Spine. 2000; 25(24):3186-91.

22. Mesquita AR, Santos EA, Porto JG, Barros IMC, Lyra DP. Translation in Brazilian portuguese and content validation of the instrument "avaliação do processo de atendimento farmacêutico". Lat Am J Pharm. 2012; 31(10):1422-9.

23. Puggina AC, Silva MJP. Validação e adaptação cultural para o português da Interpersonal Communication Competence Scale. Acta Paul Enferm. 2014; 27(2):108-14.

24. Amaral ABCN, Rider EA, Lajolo PP, Tone LG, Pinto RMC, Lajolo MP, et al. Development of a Brazilian portuguese adapted version of the Gap-Kalamazoo communication skills assessment form. Int J Med Educ. 2016; 7:400-5. 
25. Pereira TJ, Puggina AC. Validation of the self-assessment of communication skills and professionalism for nurses. Rev Bras Enferm. 2017; 70(3):588-94.

26. Costa DAS, Silva RF, Lima VV, Ribeiro ECO. Diretrizes curriculares nacionais das profissões da Saúde 2001-2004: análise à luz das teorias de desenvolvimento curricular. Interface (Botucatu). 2018; 22(67):1183-95.

27. Vasconcelos MFF, Nicolotti CA, Silva JF, Pereira SMLR. Entre políticas (EPS Educação Permanente em Saúde e PNH - Política Nacional de Humanização): por um modo de formar no/para o Sistema Único de Saúde (SUS). Interface (Botucatu). 2016; 20(59):981-91.

28. Mattos RA. Princípios do Sistema Único de Saúde (SUS) e a humanização das práticas de saúde. Interface (Botucatu). 2009; 13 Suppl 1:771-80.

29. Cömert M, Zill JM, Christalle E, Dirmaier J, Härter M, Scholl I. Assessing communication skills of medical students in objective structured clinical examinations (OSCE) - A systematic review of rating scales. PLoS One. 2016; 11(3):e0152717.

30. Boon H, Stewart M. Patient-physician communication assessment instruments:1986 to 1996 in review. Patient Educ Couns. 1998; 35(3):161-76.

31. Brouwers M, Rasenberg E, van Weel C, Laan R, van Weel-Baumgarten E. Assessing patient-centred communication in teaching: a systematic review of instruments. Med Educ. 2017; 51(11):1103-17.

32. Epstein J, Santo RM, Guillemin F. A review of guidelines for cross-cultural adaptation of questionnaires could not bring out a consensus. J Clin Epidemiol. 2015; 68(4):435-41.

33. Felisbino MB, Steidle LJM, Gonçalves-Tavares M, Pizzichini MMM, Pizzichini E. Questionário de Leicester sobre tosse crônica: tradução e adaptação cultural para a língua portuguesa falada no Brasil. J Bras Pneumol. 2014; 40(3):213-21.

34. Fjortoft N. Self-assessment in pharmacy education. Am J Pharm Educ. 2006; 70(3):64.

35. Lundquist LM, Shogbon AO, Momary KM, Rogers HK. A comparison of students' self-assessments with faculty evaluations of their communication skills. Am J Pharm Educ. 2013; 77(4):72.

36. Donnon T, Al Ansari A, Al Alawi S, Violato C. The reliability, validity, and feasibility of multisource feedback physician assessment. Acad Med. 2014; 89(3):511-6.

37. Galato D, Alano GM, França TF, Vieira AC. Exame clínico objetivo estruturado (ECOE): uma experiência de ensino por meio de simulação do atendimento farmacêutico. Interface (Botucatu). 2011; 15(36):309-19.

38. Miranda FBG, Mazzo A, Pereira Junior GA. Avaliação de competências individuais e interprofissionais de profissionais de saúde em atividades clínicas simuladas: scoping review. Interface (Botucatu). 2018; 22(67):1221-34.

39. Rehim SA, DeMoor S, Olmsted R, Dent DL, Parker-Raley J. Tools for assessment of communication skills of hospital action teams: a systematic review. J Surg Educ. 2017; $74(2): 341-51$.

40. Raymundo VP. Construção e validação de instrumentos: um desafio para a psicolinguística. Let Hoje. 2009; 44(3):86-93.

41. Pasquali L. Instrumentação psicológica: fundamentos e práticas. Porto Alegre: Artmed; 2009. 
Teaching communication skills to health professionals is extremely important. This study aimed to identify instruments validated for Brazilian Portuguese that assess communication skills of health students or professionals. We carried out a scoping review that included validation studies of instruments for assessing communication skills of students/professionals from the area of Health. Four instruments were identified, translated into Brazilian Portuguese and validated for this language. Content validation, construct validation and the internal consistency test were the most frequently performed ones. This review identified a reduced number of instruments to assess communication skills and limited data about the instruments' validity and reliability.

Keywords: Validation studies. Communication skills. Healthcare.

La enseñanza de habilidades de comunicación es indispensable para los profesionales de la salud. El objetivo de este estudio fue identificar instrumentos validados en portugués de Brasil que evalúan habilidades de comunicación de estudiantes o profesionales de la salud. Para ello, se realizó una revisión de alcance que incluyó estudios de validación de instrumentos para evaluación de habilidades de comunicación de estudiantes/profesionales del área de la salud. Se identificaron cuatro instrumentos, traducidos y validados al portugués de Brasil. La validación de contenido, la validación del constructo y el test de consistencia interna se realizaron con mayor frecuencia. Esta revisión identificó un número reducido de instrumentos para evaluar habilidades de comunicación y datos limitados de validez y confiabilidad de los instrumentos.

Palabras clave: Estudios de validación. Habilidades de comunicación. Cuidado de salud. 